\title{
On the suitability of high vacuum electrospray deposition for the fabrication of molecular electronic devices
}

\author{
Robert H. Temperton ${ }^{\mathrm{a}}$, James N. O'Shea ${ }^{\mathrm{a}}$, David J. Scurr ${ }^{\mathrm{b}}$ \\ ${ }^{a}$ School of Physics, University of Nottingham, Nottingham NG7 2RD, UK \\ ${ }^{b}$ School of Pharmacy, University of Nottingham, Nottingham NG7 2RD, UK
}

\begin{abstract}
We present a series of three studies investigating the potential application of high vacuum electrospray deposition to construct molecular electronic devices. Through the use of time of flight secondary ion mass spectrometry we explore the use of this novel deposition technique to fabricating multilayer structures using materials that are compatible with the same solvents and films containing a mixture of molecules from orthogonal solvents. Using $\mathrm{x}-$ ray photoelectron spectroscopy we study the deposition of a polymer blend using electrospray and find evidence of preferential deposition of one of the components.
\end{abstract}

Keywords: Electrospray Deposition, ToF-SIMS, XPS, Molecular Electronics, Polymers

\section{Introduction}

Vacuum electrospray deposition (ESD) is an emerging technique that has allowed researchers to study complex molecules on surfaces, in-situ, using a vast array of analytical techniques requiring high vacuum including various photoemission [1, 2, 3] and scanning probe techniques [4, 5]. Early examples of work using high vacuum ESD studied the bonding of molecules used in dye sensitised solar cells such as the benchmark N3 sensitiser dye on the titanium dioxide surface [1]. The technique has also been applied beyond

Email addresses: robert.temperton@nottingham.ac.uk (Robert H. Temperton), J.Oshea@nottingham.ac.uk (James N. O'Shea) 
traditional surface science experiments and used with biological systems 6 , 7. In recent years molecular photovoltaics, such as organic solar cells, are increasingly reliant on the construction of multilayer and bulk heterojunction structures [8, 9]. Ambient electrospray deposition has been used as a tool to construct such structures [10] providing a pathway to use high vacuum electrospray to study critical parts of such devices in-situ.

Electrospray deposition relies on the formation of a beam of molecular ions via the electrospray ionisation process. This has been thoroughly discussed elsewhere 11, 12, 13, but in summary, a fluid is passed through a small capillary tube with a large voltage $(\sim \mathrm{kV})$ applied to it. The voltage causes the liquid emerging from the capillary to be drawn into a well-defined shape known as a Taylor cone - at the tip of which the charge on the liquid is so high a jet of liquid is expelled. Due to the charge on the liquid's surface, the jet pinches off into a stream of droplets which in turn repeatedly fission into ever smaller drops. This plume of ionised droplets can be fired into a differentially pumped vacuum system where a series of apertures allow the pressure to be reduced to high vacuum conditions whilst skimming the ionised droplets, from which solvent is evaporating, into a narrowly diverging beam of molecular ions. Placing a surface in the path of this beam allows a film of the solute to be grown on the surface with minimal solvent contamination.

\section{Instrumentation}

The ESD system used was a Molecularspray UHV4 (a schematic is shown in Figure 1a). This portable deposition source consisted of a $250 \mu \mathrm{m}$ inlet capillary, $0.4 \mathrm{~mm}$ and $0.6 \mathrm{~mm}$ skimmer cones separating the first two vacuum stages (pumped using roughing pumps) from the 3rd vacuum stage pumped using a turbomolecular pump. The exit from the instrument was a $1 \mathrm{~mm}$ aperture. The deposition chamber, pumped using a turbomolecular pump, had a base pressure of $1 \times 10^{-7} \mathrm{mbar}$ and a deposition pressure of $1 \times 10^{-6}$ mbar. The depositions were monitored by observing fluctuations in the pressure and the inlet capillary was flushed regularly (with the valve to the deposition chamber closed) to avoid clogging/blockages from the sprayed molecules.

The solutions were fed into the emitter capillary (New Objective Stainless Steel TaperTip) by a syringe pump delivering a flow rate of $0.3 \mathrm{ml} \mathrm{h}^{-1}$. Before each deposition the emitter, syringe and tubing were thoroughly flushed with 
solvent. A bias of $\sim 2 \mathrm{kV}$ was applied to the emitter with respect to the grounded entrance capillary.

Time-of-flight secondary ion mass spectrometry (ToF-SIMS) was used as an imaging technique. ToF-SIMS utilises ion sputtering to lift fragments off the surface whilst a time of flight mass spectrometer measures mass spectra of the fragments. These spectra can be collected as a function of time and/or space in the $\mathrm{x}, \mathrm{y}$ plane of the sample. This allows chemical maps of the sample to be constructed, in which each pixel represents a full mass spectrum, making it the ideal technique for imaging sub millimetre structures with chemical contrast. The instrument used was an ToF-SIMS IV instrument (IONTOF $\mathrm{GmbH}$ ) with a $25 \mathrm{keV}$ Bi primary ion source (with a $\mathrm{Bi}_{3}^{+}$cluster beam applied). The primary ion dose was kept below the static limit and an electron flood gun $(<20 \mathrm{eV})$ was used to prevent charging.

X-ray photoelectron spectroscopy (XPS) was used to provide chemical analysis of thin films and bulk samples. By irradiating a sample with $\mathrm{x}$ ray photons, electrons are emitted from the sample into the vacuum (the photoelectric effect). By measuring the kinetic energy of these emitted photoelectrons, the original binding energy of the electrons can be determined meaning XPS is sensitive to the chemical environment of electrons. XPS therefore allows quantitative measurements of blends of molecules that may contain the same elements existing in different chemical states, and due to its high surface sensitivity (limited to the nanometre range by the escape depth of the photoelectrons), XPS is ideal for studying the chemistry of thin films. In this study, a Kratos Axis Ultra instrument was used with a monochromotised aluminium $K \alpha$ x-ray source. Charge neutralisation was provided using an electron flood gun. The analyser was set to measure in hybrid mode with a pass energy of $20 \mathrm{eV}$.

The molecules and solutions used for each of the three experiments are detailed in their respective sections. All were purchased from Sigma Aldrich.

\section{Methods, results and discussion}

\subsection{Layered deposits of molecules from the same solvent}

Multilayer structures of different organic materials are critical in the construction of a range of devices including Bragg reflectors 14], photovoltaic devices such as organic solar cells [8], and drug delivery systems [15]. Techniques like spin casting provide the capability to build high quality multilayer 

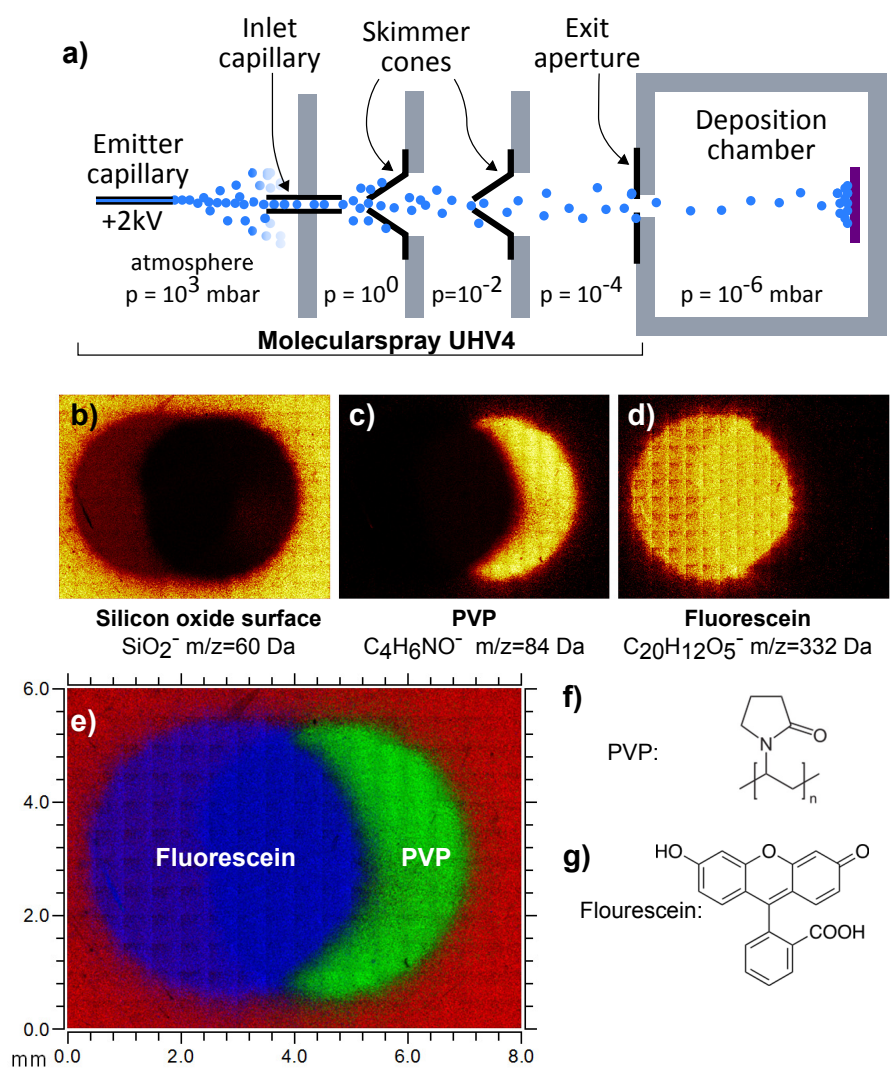

Figure 1: a) Schematic of the Molecularspray UHV4 system used to fabricate bi-layer structures. b-d) ToF-SIMS images built using markers for $\mathrm{SiO}_{2}, \mathrm{PVP}$ and fluorescein respectively. e) Composite image using the above 3 markers shown in red, green and blue respectively. f-g) chemical structure of PVP and fluorescein.

structures from molecules that are compatible with different solvents: Bailey et al have shown, using ToF-SIMS, that alternate spin cast layers of polystyrene (PS) and polyvinylpyrrolidone (PVP) dissolved in toluene and water respectively, can produce multilayer structures that have highly repeatable layer thickness and sharp interfaces [16]. However, these traditional wet chemistry preparation methods are limited to the use of materials dissolved in orthogonal solvents (solvents that will dissolve one species but not another). Solvent free techniques, such as electrospray deposition, provide a potential solution to overcoming this experimental limitation.

A silicon wafer substrate was cleaned via ultrasonication in acetone, methanol and isopropanol. Thin films of polyvinylpyrrolidone (PVP), with 
an average molecular weight $360 \mathrm{kDa}$, followed by fluorescein dye were deposited at room temperature using electrospray onto the native oxide surface. Both molecules were dissolved in methanol to a concentration of $0.1 \%$ (w.t) and further diluted to $0.05 \%$ (w.t) for deposition. Each deposition lasted an hour and the sample, which was held on a transfer arm $0.5 \mathrm{~m}$ away from the exit aperture of the UHV4, was moved slightly between depositions; both depositions were therefore visible but with an overlapping area.

ToF-SIMs was used to image this fluorescein/PVP bi-layer on the silicon oxide surface. Figure 1 $1 \mathrm{~b}-\mathrm{d}$ shows secondary ion images constructed from the intensity of individual peaks in the mass spectra selected as markers for the surface and each molecule. The spectral values used as markers for the oxide surface, PVP and fluorescein were $60 \mathrm{Da}, 332 \mathrm{Da}$ and $84 \mathrm{Da}$. A composite image showing the intensity of the different markers is also included in Figure 1․ These images show there is minimal mixing between the layers where the fluorescein film appears on top of the PVP deposit eclipsing the image of the PVP (Figure 1k) with little signal attributed to the PVP in the overlapping region. Although, the depositions were very thin, some preliminary depth profiling experiments illustrated the presence of underlying PVP in the overlapping region (data not shown).

\subsection{Deposition of immiscible polymer blends}

Producing a film containing a blend of molecules from orthogonal solvents can be an experimentally challenging problem. Electrospray deposition provides a potential solution; as there should be minimal amounts of solvent, simultaneous depositions of the two molecules from different electrospray sources should allow the mixing of the molecules at the surface. This was attempted using the setup shown in Figure 2a. Two electrospray sources, of the type used in section 3.1, were placed at $90^{\circ}$ to each other with the sample at $45^{\circ}$. The sources were $8 \mathrm{~cm}$ from the surface. It was not possible to monitor the pressure in the chamber during deposition but the system was allowed to pump down sufficiently that the ultimate deposition pressure of $\sim 1 \times 10^{-6}$ mbar was achieved. 


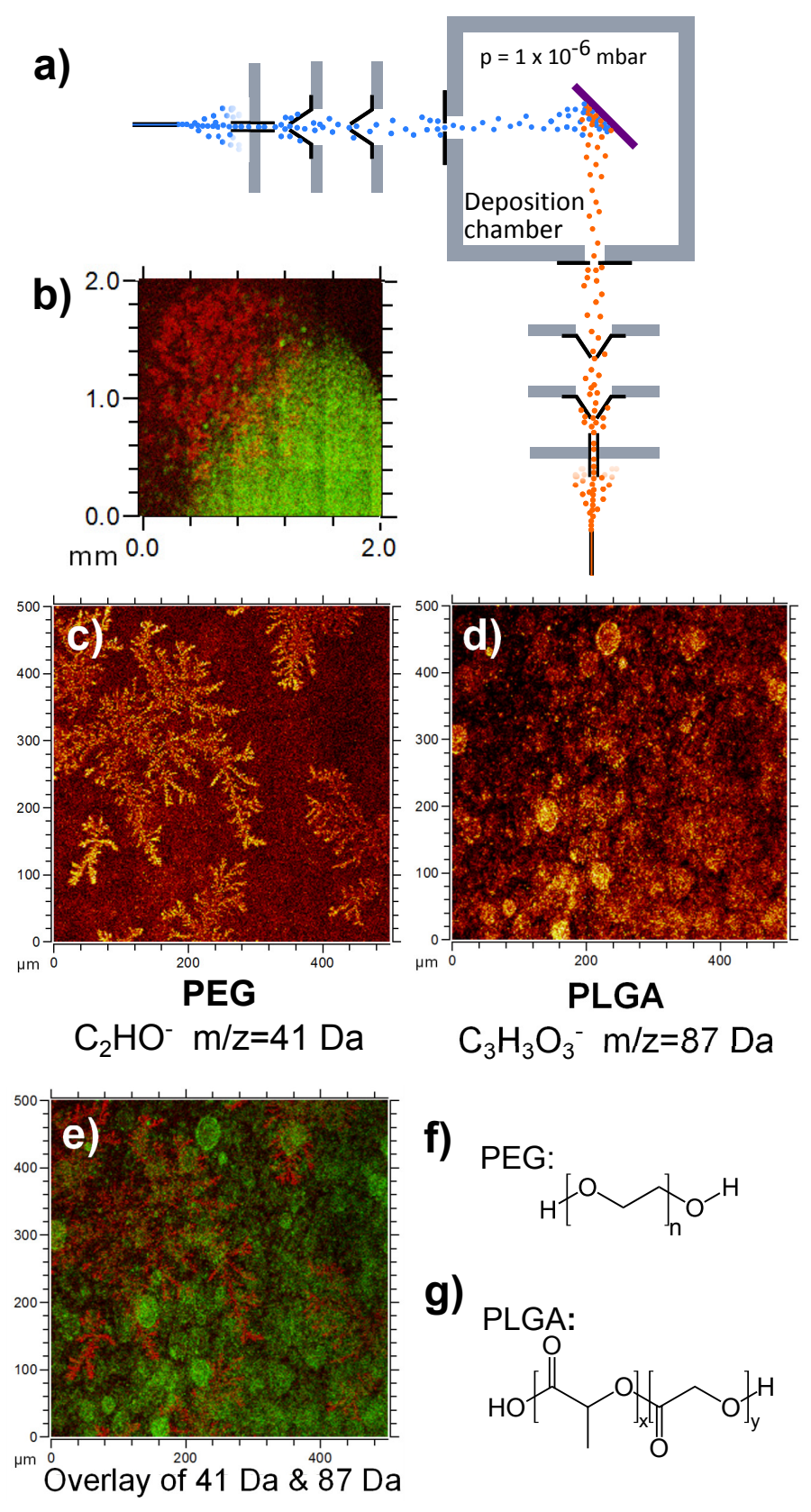

Figure 2: a) Geometry used to co-deposit molecules from two solutions using two Molecularspray UHV4 systems. b) ToF-SIMS composite image showing the markers for PEG (red) and PLGA (green). c-e) high resolution images of the overlap region for each of the two markers a composite respectively. f-g) show the chemical structure of PEG and PLGA. 
The two polymers used were poly(lactic-co-glycolic acid) (PLGA) and Polyethylene glycol (PEG) dissolved in acetone and methanol respectively to a concentration of $0.1 \%$ (w.t.). The PLGA, a co-polymer with average molecular weight between $40 \mathrm{kDa}$ and $75 \mathrm{kDa}$, had a ratio of $65: 35$ for the lactide:glycolide monomer components. The PEG had a molecular weight of $3.35 \mathrm{kDa}$. PLGA and PEG were co-deposited at room temperature for around 1 hour onto a clean silicon wafer (ultrasonicated in acetone, methanol and isopropanol) and the resulting deposition was imaged using ToF-SIMS. A sample of the images collected are shown in Figure 2. The two diagnostic secondary ions used are $m / z=41\left(\mathrm{C}_{2} \mathrm{HO}^{-}\right)$for PEG and $m / z=87$ $\left(\mathrm{C}_{3} \mathrm{H}_{3} \mathrm{O}_{3}^{-}\right)$for PLGA. A $2 \mathrm{~mm}$ area overlay image is shown in Figure $2 \mathrm{~b}$ illustrating the overlapping deposits. A $500 \mu \mathrm{m}$ area within the overlap region is shown for the PEG and PLGA markers and as an overlay in Figures $2 \mathrm{c}$ $2 \mathrm{~d}$ and $2 \mathrm{p}$ respectively. Secondary ion images were also measured away from the overlapping region in areas containing each individual species. The morphologies of which showed comparable structure to that of the overlapping region.

Firstly it is clear that in the overlapping region there has been some mixing of the two components. The PEG has also formed a large scale fractal structure on the surface. This behaviour implies the molecule is mobile on the surface, which, considering the size of the PEG polymer chains, implies there is likely a small amount of solvent on the surface allowing such structures to form. The same argument could also be made for the "blob" like structure of the PLGA. Despite this, an immiscible polymer blend has been achieved on the surface with domain sizes on the micron length scale.

Although acetone and methanol are miscible, when the two solutions were mixed, the mixture turned cloudy as the molecules partially drop out of solution. The same experiment does however have the potential to be applied to completely immiscible polymer solutions, for example those soluble exclusively in water and toluene.

\subsection{Miscible polymer blends for organic photovoltaics}

The polymer blend PEDOT:PSS is used in organic photovoltaic devices as a hole conducting layer. It contains a mixture of poly(3,4-ethylenedioxythiophene) and polystyrene sulfonate as shown in Figure $3 \mathrm{~d}$ and 3 . Here we use XPS to compare films of PEDOT:PSS deposited using electrospray deposition and the more traditional spin casting directly from solution. 
a)

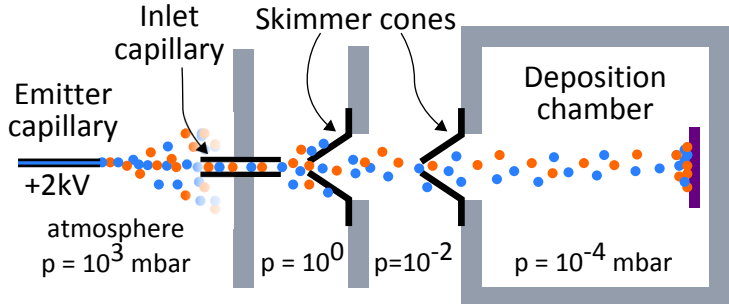

b)

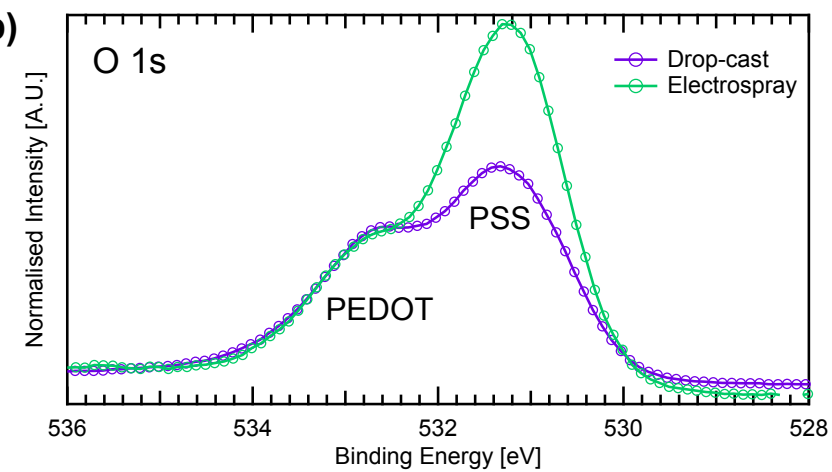

c)
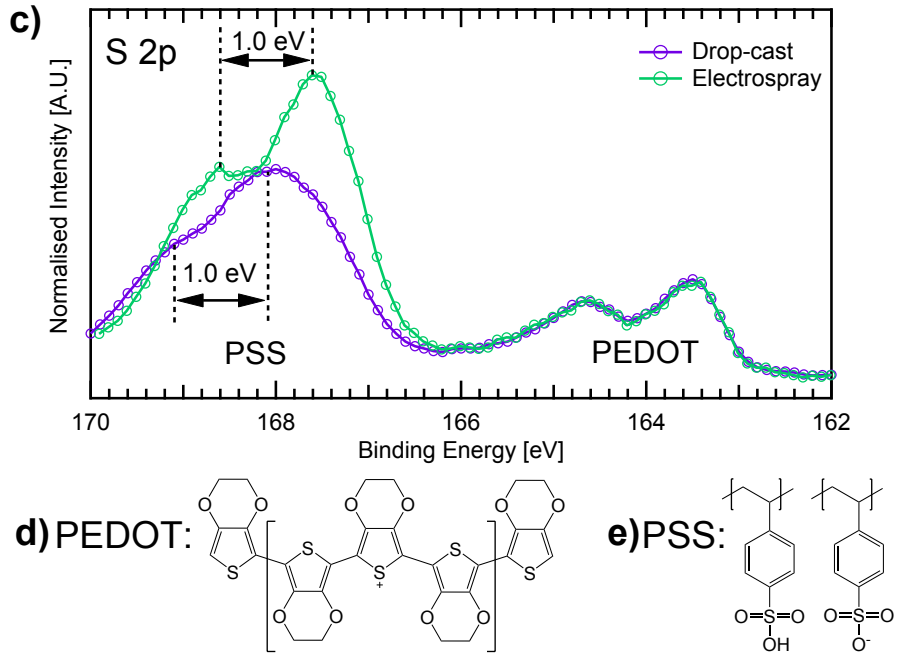

Figure 3: a) The electrospray deposition source used to deposit PEDOT:PSS consisted of one less pumping stage than the UHV4 source used in the previous sections. b-c) show O $1 s$ and S $2 p$ XPS measurements for an electrospray deposited sample and a drop cast sample of the same solution. d-e) show the two components of the PEDOT:PSS polymer blend. PSS is a co-polymer of the two groups shown. 
Gold substrates were made by thermally evaporating $10 \mathrm{~nm}$ of titanium and $100 \mathrm{~nm}$ gold onto silicon wafer. The titanium was used to improve cohesion between the gold film and the silicon oxide surface. The silicon wafer was cleaned by ultrasonication in acetone, methanol and isopropanol prior to evaporation. PEDOT:PSS (Orgacon dry re-dispersible pellets by Agfa) was dissolved in deionised water and drop cast onto one substrate. This solution was diluted to $0.01 \%$ (w.t.) and electrosprayed onto another substrate using the apparatus shown in Figure 3 a . Both experiments were carried out with the substrates at room temperature. This apparatus contained one fewer pumping stage than the Molecularspray UHV4 (as used in Sections 3.1 and 3.2) to maximise deposition flux as low pressures and desolvation were less critical. $10 \%$ methanol added to the solution to improve the quality of the spray.

Figures $3 \mathrm{~b}$ shows XPS measurements of the O $1 \mathrm{~s}$ region for both the drop cast and electrospray deposition samples (both were prepared ex-situ). The $\mathrm{O} 1 \mathrm{~s}$ spectra contains two overlapping peaks with binding energies of $532.8 \mathrm{eV}$ and $531.3 \mathrm{eV}$. We have attributed the higher binding energy peak to the ethylenedioxy attachment to the thiophene ring of the PEDOT and the lower binding energy peak to the sulfonate side group of the PSS. Both spectra have been normalised to the PEDOT/thiophene shoulder. There is a clear increase of the PSS/sulfonate contribution for the electrosprayed sample.

Figure $3 c$ shows XPS spectra of the $\mathrm{S} 2 p$ region (measured at the same position on both samples as the $\mathrm{O} 1 s$ data). There are two doublets in the spectra resulting from the spin orbit splitting of the $2 p$ states of sulphur in the PEDOT and PSS molecules. We have attributed the lower binding energy doublet, centred at approximately $164 \mathrm{eV}$, to the PEDOT's thiophene ring and the much broader feature at higher binding energy, centred at approximately $168 \mathrm{eV}$, to the sulfonate group of the PSS. Again, both spectra are normalised to the PEDOT contribution and there is a clear increase in PSS signal for the electrosprayed sample.

The broadening and binding energy shift of the sulfonate peaks of the drop cast relative to the ESD sample is attributed to charging effects due to the thickness of the drop cast deposit. We suspect that this is only present in the PSS and not the PEDOT as the PEDOT is a fully conjugated conductive polymer. Measurement at different sample positions shows the same feature shapes and there was no sign of beam damage between the start and end measurements at a single position. 
It is worth noting that the two polymers in the PEDOT:PSS blend are individually charged. As shown in Figures 3d 3e, PEDOT has positive charges on the thiophene backbone whilst PSS has negatively charged sulfonate groups. It is possible this could explain why the electrospray technique would preferentially deposit one species over the other - however in this case the experiment was carried out in the positive bias mode and we are seeing potential preferential ionisation of the negative species which is counter intuitive.

Analysis of the $\mathrm{C} 1 s$ was hindered by atmospheric carbon contamination (during transport of the samples from the deposition apparatus to the XPS instrument) so is not included here. Carbon spectra were used to provide relative calibration between the two samples where the low binding energy edge of the $\mathrm{C} 1 s$ region implies a $0.9 \mathrm{eV}$ shift between the two samples. This offset was applied to all the included spectra.

\section{Summary and conclusions}

Three experiments have been presented highlighting the capabilities and potential challenges of using electrospray to produce multi-layered structures and blends of materials. Firstly we have shown that the solvent contamination on the surface is low enough to build up thin layers of molecules that are compatible with the same solvents without substantial mixing of the films. However, ultra high vacuum compatible electrospray deposition is not well suited to building up thick deposits and although SIMS is surface sensitive enough to measure surface coverage, conventional sputtering does not allow depth profiling of such thin films (when probed with XPS we find similar deposits are several monolayers thick). With the co-deposition experiment we have shown there is almost certainly a small solvent contribution to the deposit allowing structure to form on the surface. More sophisticated electrospray instruments have applied techniques such as mass selection and heated inlet capillaries to attempt to combat these issues.

The XPS analysis of the PEDOT:PSS blend is of importance and certainly needs further study. It appears as though the electrospray deposition process preferentially ends up with one species over another being ionised, entering the vacuum system and landing on the surface. Although we have shown with SIMS that a simple vacuum compatible electrospray source can be utilised to build the sort of films used in molecular devices, care should be taken if simultaneously depositing multiple species. 


\section{Acknowledgements}

The authors are grateful for technical support from Emily Smith at Nottingham's Nanoscale and Microscale Research Centre (NMRC) XPS facility. Equipment access was funded by University of Nottingham Interdisciplinary Center for Analytical Science (UNICAS) and Advanced Molecular Materials Research Priority Area. Further funding was provided by EPSRC and Molecularspray Ltd through a DTG/CASE conversion studentship, and a Nottingham University discipline bridging award.

[1] L. C. Mayor, J. Ben Taylor, G. Magnano, A. Rienzo, C. J. Satterley, J. N. O'Shea, J. Schnadt, Photoemission, resonant photoemission, and x-ray absorption of a $\mathrm{Ru}(\mathrm{II})$ complex adsorbed on rutile $\mathrm{TiO} 2(110)$ prepared by in situ electrospray deposition., The Journal of chemical physics 129 (2008) 114701.

[2] A. J. Britton, M. Weston, J. B. Taylor, A. Rienzo, L. C. Mayor, J. N. O'Shea, Charge transfer interactions of a $\mathrm{Ru}(\mathrm{II})$ dye complex and related ligand molecules adsorbed on $\mathrm{Au}(111)$., The Journal of chemical physics 135 (2011) 164702.

[3] A. J. Gibson, R. H. Temperton, K. Handrup, M. Weston, L. C. Mayor, J. N. O'Shea, Charge transfer from an adsorbed ruthenium-based photosensitizer through an ultra-thin aluminium oxide layer and into a metallic substrate., The Journal of chemical physics 140 (2014) 234708.

[4] A. Saywell, J. K. Sprafke, L. J. Esdaile, A. J. Britton, A. Rienzo, H. L. Anderson, J. N. O'Shea, P. H. Beton, Conformation and packing of porphyrin polymer chains deposited using electrospray on a gold surface., Angewandte Chemie (International ed. in English) 49 (2010) 9136-9.

[5] C. J. Satterley, L. M. a. Perdigão, A. Saywell, G. Magnano, A. Rienzo, L. C. Mayor, V. R. Dhanak, P. H. Beton, J. N. O'Shea, Electrospray deposition of fullerenes in ultra-high vacuum: in situ scanning tunneling microscopy and photoemission spectroscopy, Nanotechnology 18 (2007) 455304 .

[6] E. Fornari, C. J. Roberts, R. H. Temperton, J. N. O'Shea, Electrospray deposition in vacuum as method to create functionally active protein immobilization on polymeric substrates., Journal of colloid and interface science 453 (2015) 252-9. 
[7] S. Rauschenbach, F. L. Stadler, E. Lunedei, N. Malinowski, S. Koltsov, G. Costantini, K. Kern, Electrospray ion beam deposition of clusters and biomolecules., Small (Weinheim an der Bergstrasse, Germany) 2 (2006) 540-7.

[8] B. Kippelen, J.-L. Brédas, Organic photovoltaics, Energy \& Environmental Science 2 (2009) 251.

[9] F. Deschler, D. Riedel, B. Ecker, E. von Hauff, E. Da Como, R. C. I. MacKenzie, Increasing organic solar cell efficiency with polymer interlayers, Phys. Chem. Chem. Phys. 15 (2013) 764-769.

[10] T. Fukuda, K. Takagi, T. Asano, Z. Honda, N. Kamata, K. Ueno, H. Shirai, J. Ju, Y. Yamagata, Y. Tajima, Bulk heterojunction organic photovoltaic cell fabricated by the electrospray deposition method using mixed organic solvent, Physica Status Solidi-Rapid Research Letters 5 (2011) 229-231.

[11] P. Kebarle, U. H. Verkerk, Electrospray: from ions in solution to ions in the gas phase, what we know now., Mass spectrometry reviews 28 (2009) 898-917.

[12] S. Rauschenbach, R. Vogelgesang, N. Malinowski, J. W. Gerlach, M. Benyoucef, G. Costantini, Z. Deng, N. Thontasen, K. Kern, Electrospray ion beam deposition: soft-landing and fragmentation of functional molecules at solid surfaces., ACS nano 3 (2009) 2901-10.

[13] J. C. Swarbrick, J. B. Taylor, J. N. O'Shea, Electrospray deposition in vacuum, Applied Surface Science 252 (2006) 5622-5626.

[14] J. Bailey, J. S. Sharp, Thin film polymer photonics: Spin cast distributed Bragg reflectors and chirped polymer structures, European Physical Journal E 33 (2010) 41-49.

[15] C. E. Mora-Huertas, H. Fessi, A. Elaissari, Polymer-based nanocapsules for drug delivery, International Journal of Pharmaceutics 385 (2010) $113-142$.

[16] J. Bailey, R. Havelund, A. G. Shard, I. S. Gilmore, M. R. Alexander, J. S. Sharp, D. J. Scurr, 3D ToF-SIMS imaging of polymer multilayer 
297 films using argon cluster sputter depth profiling, ACS Applied Materials and Interfaces 7 (2015) 2654-2659. 